\title{
Friedel oscillations in a gas of interacting one-dimensional fermionic atoms confined in a harmonic trap
}

\author{
S N Artemenko†, Gao Xianlong $\ddagger$, and W Wonneberger $\ddagger$ \\ $\dagger$ Institute of Radio Engineering and Electronics, Moscow 125009, Russia \\ $\ddagger$ Abteilung Mathematische Physik, Universität Ulm, D89069 Ulm, Germany
}

\begin{abstract}
Using an asymptotic phase representation of the particle density operator $\hat{\rho}(z)$ in the one-dimensional harmonic trap, the part $\delta \hat{\rho}_{F}(z)$ which describes the Friedel oscillations is extracted. The expectation value $\left\langle\delta \hat{\rho}_{F}(z)\right\rangle$ with respect to the interacting ground state requires the calculation of the mean square average of a properly defined phase operator. This calculation is performed analytically for the Tomonaga-Luttinger model with harmonic confinement. It is found that the envelope of the Friedel oscillations at zero temperature decays with the boundary exponent $\nu=(K+1) / 2$ away from the classical boundaries. This value differs from that known for open boundary conditions or strong pinning impurities. The soft boundary in the present case thus modifies the decay of Friedel oscillations. The case of two components is also discussed.
\end{abstract}

PACS numbers: 71.10.Pm, 05.30.Fk, 03.75.Ss

Short title: Friedel oscillations in a harmonic trap

October 30, 2018 


\section{Introduction}

Recent experimental successes in obtaining degeneracy in three-dimensional ultracold Fermi vapors [1-7], possibly in combination with microtrap technology [8-13], make it conceivable to realize the quasi one-dimensional neutral Fermi gas confined in a trapping potential without the complications due to contacts and impurities.

Friedel oscillations are a principal feature of a degenerate Fermi gas when translational invariance is broken. Usually, impurities [14] are the cause. However, boundaries can also be responsible for Friedel oscillations. The spatial period of Friedel oscillations is $\lambda_{F}=\pi / k_{F}$, where $k_{F}$ is the Fermi wave number. This effect is particularly pronounced in one spatial dimension because then the susceptibility becomes logarithmically singular at $2 k_{F}$ due to perfect nesting.

It is known from the theory of one-dimensional fermions confined between hard walls (bounded Luttinger liquids $=$ BLL) that interactions modify the decay of Friedel oscillation away from the boundary [15-17].

In this article, we investigate the Friedel oscillations at zero temperature both for the spin-polarized one component system, when s-wave scattering is forbidden, as well as for the two component system. We apply a recent model of $N \gg 1$ interacting fermionic atoms in one spatial dimension confined in a harmonic trap [18]. The model which can be termed "Tomonaga-Luttinger model with harmonic confinement" is analytically solvable in a similar way as the Luttinger model (cf. e.g. [19-22]) using bosonization. The present model is simpler than the Luttinger model in that it has only one (nonchiral) branch in contrast to the two chiral branches of the latter which result from an artificial split of an otherwise continuous band.

It is found that near the classical boundaries, Friedel oscillations decay in a way which differs from the known result for BLL [15-17]. It also differs from the result for an infinitely strong pinning impurity [23] which acts as an invariant hard wall under scaling [24].

The calculations become possible because the fermion density in the harmonic trap can be decomposed asymptotically (i.e., for $N \gg 1$ ) into a slowly varying part and a part describing the Friedel oscillations. Both parts involve a specific phase operator for which a free field theory is available.

Friedel oscillations in realistic Fermi gas are difficult to observe experimentally at least for two reasons: the integrated total mass in the one-dimensional Friedeloscillations is of the order of one atom (cf. e.g. [25]) though repulsive interactions increase their weight. Furthermore, temperature effects blur the oscillations unless $k_{B} T<\hbar \omega_{\ell}$ where $\omega_{\ell}$ is the longitudinal trap frequency [26]. Provided these exceedingly low temperatures can be realized, we can adopt the argument in [25]: it is conceivable to use an array of short micro traps each filled with a reduced number of atoms (thus 
avoiding instabilities). The oscillations within each trap then add up and lead to a total effect that is enhanced by the number of traps. Using micro fabrication techniques it should be possible to combine 100 traps on one substrate leading to a signal that may become within reach of advanced imaging techniques.

\section{Theoretical framework}

\subsection{Tomonaga-Luttinger model with harmonic confinement}

Here, we give a short review of the model which is used for the present calculation. More details are given in [18].

We start from an effective pair interaction

$$
\hat{V}=\frac{1}{2} \sum_{m n p q} V(m, p ; q, n)\left(\hat{c}_{m}^{+} \hat{c}_{q}\right)\left(\hat{c}_{p}^{+} \hat{c}_{n}\right)
$$

between one-dimensional spin polarized fermions. The fermionic creation and destruction operators $\hat{c}_{m}^{+}$and $\hat{c}_{q}$ are taken in the basis of harmonic oscillator wave functions. Thus the harmonic trap topology is exactly represented.

The Hamiltonian for interacting fermions considered here follows uniquely by retaining those parts in the fermionic pair interaction operator which are expressible in terms of density fluctuation operators $\hat{\rho}(m)=\sum_{p} \hat{c}_{p+m}^{+} \hat{c}_{p}$. These are

$$
V(m, p ; q, n) \rightarrow V_{a}(|q-m|) \delta_{m-q, n-p}+V_{b}(|q-m|) \delta_{q-m, n-p}+V_{c}(|q-p|) \delta_{m+q, n+p} .
$$

The interaction matrix elements $V_{a}(m), V_{b}(m)$, and $V_{c}(m)$ correspond to the Luttinger model coupling functions $g_{4}(p), g_{2}(p)$, and $g_{1}(p)$, respectively. $V_{a}$ and $V_{b}$ describe forward scattering and $V_{c}$ describes $2 k_{F}$ (backward) scattering. In [27], it is shown that the retained matrix elements are dominant in the limit of large $N$. This is related to approximate momentum conservation during collisions in the trap.

In the next step, the linear dispersion of free harmonic oscillator states and the addition of the anomalous vacuum is utilized to bosonize the original fermionic Hamiltonian in terms of canonically conjugate Bose operators $\hat{d}$ and $\hat{d}^{+}$in accordance with Kronig's identity [28]. This gives the bosonic form

$$
\begin{aligned}
\tilde{H}= & \frac{1}{2} \hbar \omega_{\ell} \sum_{m>0} m\left(\hat{d}_{m} \hat{d}_{m}^{+}+\hat{d}_{m}^{+} \hat{d}_{m}\right)-\frac{1}{2} \sum_{m>0} V_{c}(m) m\left(\hat{d}_{m}^{2}+\hat{d}_{m}^{+2}\right) \\
& +\frac{1}{2} \sum_{m>0} V_{c}(m) \sqrt{2 m}\left[\hat{d}_{2 m}+\hat{d}_{2 m}^{+}\right] \\
& +\frac{1}{2} \sum_{m>0} V_{a}(m) m\left(\hat{d}_{m} \hat{d}_{m}^{+}+\hat{d}_{m}^{+} \hat{d}_{m}\right)+\frac{1}{2} \sum_{m>0} V_{b}(m) m\left(\hat{d}_{m}^{2}+\hat{d}_{m}^{+2}\right)
\end{aligned}
$$


of the Hamiltonian.

Equation (3) is the version for a one-component Fermi gas, i.e., for spin polarized fermions. Usually, the remaining "p-wave" interaction is small. However, it has been demonstrated recently that Feshbach resonances can make it relevant [29].

Our simplified interaction Hamiltonian is integrable. In the sense of the Luttinger liquid phenomenology, we expect that the boundary exponent for Friedel oscillations at zero temperature and for $N \gg 1$ is invariant against details of the interaction. It is also stressed that the trap potential is exactly incorporated.

In the one-component system, backscattering dominates as demonstrated below. Accepting this, the validity of our approach could be verified analytically by perturbation theory in the fermionic Hilbert space as well as by exact numerical diagonalization of the fermionic problem [18].

\subsection{Dominance of backward scattering in the one-component gas}

The existence of only one branch results in restrictions on the values of the interaction coefficients $V_{a}$ and $V_{b}$ in the one-component case: their contribution is small in comparison to $V_{c}$ [27]. This can be demonstrated analytically by using WKB wave functions (cf. equation (21) below) in the calculation of $V(m, p ; q, n)$ : starting from an effective "p-wave" potential in one dimension

$$
V(z)=V_{p} a_{p}^{3} \partial_{z}^{2} \delta(z)
$$

one obtains for $(m, n, p, q)=O(N) \gg 1$

$$
\begin{aligned}
& V(m, p ; q, n)=\int d z d z^{\prime} \psi_{m}(z) \psi_{q}(z) V\left(z-z^{\prime}\right) \psi_{p}\left(z^{\prime}\right) \psi_{n}\left(z^{\prime}\right) \\
& =V_{p} a_{p}^{3} \int d z \psi_{m}(z) \psi_{q}(z) \partial_{z}^{2}\left\{\psi_{p}(z) \psi_{n}(z)\right\} \rightarrow \frac{4 \sqrt{2 N} \alpha^{3} a_{p}^{3} V_{p}}{\pi^{2}} F(s),
\end{aligned}
$$

with

$$
F(s) \equiv \frac{\cos ^{2}(\pi s / 2)}{s^{2}-1}, \quad s \equiv m+q-p-n
$$

Thus, each individual backscattering term in (2) belongs to the dominating $s=0$ contribution while only some terms of $V_{a, b}$-type do this. Thus backscattering dominates.

The interaction coefficient $V_{c}=V_{c}(1)$ can be expressed as 


$$
V_{c}=-\frac{2}{\pi^{2}} k_{F} a_{p}\left(\frac{V_{p}}{\frac{\hbar^{2}}{2 m_{A} a_{p}^{2}}}\right) \hbar \omega_{\ell} .
$$

\subsection{One-particle operator and phase fields}

The third contribution on the r.h.s. of equation (3) represents a one-particle operator $\hat{V}_{1}$. It originates from rearranging operators in equation (1) to bring backscattering into a bilinear form of density fluctuation operators. The one-particle operator is neglected

in the BLL as pointed out in [30]. It does not alter boundary exponents, but has quantitative effects on other properties. $\hat{V}_{1}$ is exactly taken into account in the present model.

The central dimensionless coupling constants and the renormalized level spacings for the model, equation (3), are given by [18]

$$
\begin{aligned}
K_{m} & \equiv \sqrt{\frac{\hbar \omega_{\ell}+V_{a}(m)-\left(V_{b}(m)-V_{c}(m)\right)}{\hbar \omega_{\ell}+V_{a}(m)+\left(V_{b}(m)-V_{c}(m)\right)}}, \\
\epsilon_{m} & \equiv \sqrt{\left(\hbar \omega_{\ell}+V_{a}(m)\right)^{2}-\left(V_{b}(m)-V_{c}(m)\right)^{2}},
\end{aligned}
$$

respectively.

For simplicity, the dependence of $K$ and $\epsilon$ on $m$ is occasionally suppressed. Note that $V_{a}(m) \rightarrow V_{a}$ implies that there are no interaction effects due to this matrix element in the one component theory and $V_{a}$ is strictly zero while $V_{b}$ can be neglected, i.e., the central coupling constant is

$$
K=\sqrt{\frac{\hbar \omega_{\ell}+V_{c}}{\hbar \omega_{\ell}-V_{c}}}, \quad \epsilon=\sqrt{\left(\hbar \omega_{\ell}\right)^{2}-V_{c}^{2}},
$$

with $V_{c}$ given by equation (7).

In some physical quantities, especially in the mean square phase fluctuation calculated below, the neglect of the m-dependence leads to inconsistencies because stability requires the coupling constants $K_{m}$ to approach unity for large $\mathrm{m}$ [20]. In analogy to the Luttinger model, we write approximately

$$
K_{m}=1+(K-1) \exp (-m r)
$$

An estimate for $r$ is $R / L_{F} \ll 1$ where $R$ is the spatial range of the interaction. 
Assuming the same exponential decay for the interaction matrix elements

$$
V_{c}(m)=V_{c} e^{-m r}, \quad r \ll 1,
$$

the one-particle operator can be rewritten as

$$
\hat{V}_{1}=\frac{1}{4 \pi} V_{c} \int_{-\pi}^{\pi} d u\left[\frac{e^{-r+2 i u}}{1-e^{-r+2 i u}}+\frac{e^{-r-2 i u}}{1-e^{-r-2 i u}}\right] \partial_{u} \hat{\phi}_{\text {odd }}(u),
$$

with the phase field

$\hat{\phi}_{\text {odd }}(u) \equiv \frac{1}{2}\left(\hat{\phi}(u)+\hat{\phi}^{+}(u)-\hat{\phi}(-u)-\hat{\phi}^{+}(-u)\right)=\sum_{n=1}^{\infty} \sqrt{\frac{e^{-n \eta}}{n}} \sin (n u)\left(\hat{d}_{n}+\hat{d}_{n}^{+}\right)$

and $\hat{\phi}$ is the bosonization phase operator [31],

$$
\hat{\phi}(u) \equiv-i \sum_{n=1}^{\infty} \frac{1}{\sqrt{n}} e^{i n(u+i \eta / 2)} \hat{d}_{n}
$$

In diagonalizing equation (3), the one-particle operator contributes a c-number shift which is taken care of by the new phase operator

$$
\hat{\Phi}(u)=\hat{\phi}_{\text {odd }}(u)+b(u)
$$

with

$$
b(u)=i \frac{K V_{c}}{4 \epsilon} \ln \left(\frac{1-e^{-r+2 i u}}{1-e^{-r-2 i u}}\right) .
$$

In contrast to $\hat{\phi}_{\text {odd }}$, the new phase field $\hat{\Phi}$ is homogeneous in the operators $\hat{f}$ and $\hat{f}^{+}$which diagonalize the Hamiltonian: $\hat{H}=\sum_{m} m \epsilon_{m} \hat{f}_{m}^{+} \hat{f}_{m}+$ zero mode contributions. The zero mode plays no role in the present context. The representation of the phase field $\hat{\Phi}$ in terms of the diagonalizing operators is

$$
\hat{\Phi}(u)=\sum_{m=1}^{\infty} \sqrt{\frac{K_{m}}{m}} e^{-m \eta / 2} \sin m u\left(\hat{f}_{m}+\hat{f}_{m}^{+}\right)
$$




\section{Decomposition of the density operator for the harmonic trap}

The density operator $\hat{\rho}(z)$ in Fock space for fermions in the harmonic trap is

$$
\hat{\rho}(z)=\sum_{m=0, n=0}^{\infty} \psi_{m}(z) \psi_{n}(z) \hat{c}_{m}^{+} \hat{c}_{n}
$$

Bosonizing $\hat{\rho}(z)$ using the auxiliary field method in [31], i.e.,

$$
\begin{aligned}
\hat{c}_{m}^{+} \hat{c}_{n}= & \int_{0}^{2 \pi} \int_{0}^{2 \pi} \frac{d u d v}{4 \pi^{2}} e^{i(m u-n v)} \frac{e^{-i(N-1)(u-v)}}{1-e^{-\eta+i(u-v)}} \\
& \times \exp \left\{-i\left(\hat{\phi}^{+}(u)-\hat{\phi}^{+}(v)\right)\right\} \exp \{-i(\hat{\phi}(u)-\hat{\phi}(v))\}
\end{aligned}
$$

implies a projection onto the subspace of $N$ fermions: $\hat{\rho}(z) \rightarrow \hat{\rho}_{N}(z)$.

We will extract from $\hat{\rho}_{N}(z)$ a slowly varying part and a part associated with the Friedel oscillations.

After bosonization, $\hat{\rho}_{N}(z)$ is given by

$$
\begin{array}{r}
\hat{\rho}_{N}(z)=\int_{-\pi}^{\pi} \frac{d u d v}{4 \pi^{2}} \frac{e^{-i(N-1)(u-v)}}{1-e^{-\eta+i(u-v)}} e^{-i \hat{\phi}^{+}(u)+i \hat{\phi}^{+}(v)} e^{-i \hat{\phi}(u)+i \hat{\phi}(v)} \\
\times\left[\sum_{m=0, n=0}^{\infty} \psi_{m}(z) \psi_{n}(z) e^{i m u-i n v}\right] .
\end{array}
$$

Here, $\eta$ is a positive infinitesimal.

Using the form factor $Z(z)=\sqrt{1-z^{2} / L_{F}^{2}}$ of the harmonic trap, where $2 L_{F}=$ $2 \ell \sqrt{2 N-1}$ is the semi-classical extension of the Fermi sea, the harmonic oscillator wave functions $\psi_{m}$ can be approximated for $N \gg 1$ by their WKB form $(\alpha=1 / \ell, \ell$ : oscillator length) according to

$$
\psi_{m}(z) \rightarrow\left(\frac{2 \alpha^{2}}{\pi^{2} m Z^{2}(z)}\right)^{1 / 4} \cos \left(\int_{0}^{z} d x k_{m}(x)-\frac{\pi m}{2}\right) .
$$

Because of the rapidly oscillating phase factor $\exp (-i(N-1)(u-v))$, an expansion around the Fermi edge with

$$
k_{m}(z)=\alpha \sqrt{2 m+1-\alpha^{2} z^{2}} \approx k_{F} Z(z)+\frac{\tilde{m}}{L_{F} Z(z)}, k_{F}=\alpha \sqrt{2 N-1}, m=N-1+\tilde{m}(22)
$$


and

$$
\begin{aligned}
\int_{0}^{z} d x k_{m}(x) & \approx \frac{k_{F}}{2}\left\{z Z(z)+L_{F} \arcsin \left(\frac{z}{L_{F}}\right)\right\}+\tilde{m} \arcsin \left(\frac{z}{L_{F}}\right) \\
& \equiv k_{F} \tilde{z}(z)+\tilde{m} \arcsin \left(\frac{z}{L_{F}}\right)
\end{aligned}
$$

is reasonable because it will compensate that factor. Explicitly, $\tilde{z}(z)$ is given by

$2 k_{F} \tilde{z}(z)=k_{F} Z(z) z+k_{F} L_{F} \arcsin \frac{z}{L_{F}}=k_{F} Z(z) z+(2 N-1) \arcsin \frac{z}{L_{F}}$.

We consider the sum over $m$ in equation (20). Extending the $\tilde{m}$-summation to $-\infty$ and setting $m=N$ in phase insensitive terms, leads to the asymptotic expansion

$$
\begin{aligned}
& \sum_{m=0}^{\infty} \psi_{m}(z) e^{i m u} \rightarrow\left(\frac{2 \pi^{2} \alpha^{2}}{N Z^{2}(z)}\right)^{1 / 4} e^{i(N-1) u} \\
& \times\left\{e^{i k_{F} \tilde{z}(z)-i \pi(N-1) / 2}\left(1-i \frac{\partial_{u}}{N}\right)^{-1 / 4} \delta\left(u+u_{0}(z)\right)\right. \\
& \left.+e^{-i k_{F} \tilde{z}(z)+i \pi(N-1) / 2}\left(1-i \frac{\partial_{u}}{N}\right)^{-1 / 4} \delta\left(u-u_{0}(z)\right)\right\} .
\end{aligned}
$$

It is noted that the core states are not properly represented in the expansion (25). As a consequence, one does not obtain correct information about the operator for the average density. Thus we will retain only the fluctuating part $\hat{\rho}_{N} \rightarrow \delta \hat{\rho}$ which is of main interest here.

Applying this expansion to equation (20), we find to lowest order in $\partial_{u} / N$

$$
\delta \hat{\rho}(z)=\frac{1}{\pi} \partial_{z} \hat{\phi}_{\text {odd }}\left(u_{0}(z)\right)-\frac{(-1)^{N}}{\pi \eta L_{F} Z(z)} \cos 2\left[k_{F} \tilde{z}(z)+\hat{\phi}_{\text {odd }}\left(u_{0}(z)\right)\right] .
$$

The phase field $\hat{\phi}_{\text {odd }}(u)$ is found to be identical to that of equation (13). This phase operator $\hat{\phi}_{\text {odd }}$ plays a central role in the present investigation.

The density operator (26) consists of two parts involving the phase operator $\hat{\phi}_{\text {odd }}$, a gradient term for slow spatial density variations and a rapidly oscillating term $\delta \hat{\rho}_{F}$ describing the Friedel oscillations. This structure is well known from the theory of the Luttinger model. In the latter case, the argument of the phase operator is the spatial 
coordinate. In the present case, however, a non-linear relation between the spatial coordinate $z$ and the variable $u_{0}(z)$ in the phase operator according to

$$
u_{0}(z)=\arcsin \left(\frac{z}{L_{F}}\right)-\frac{\pi}{2}
$$

appears which reflects the harmonic trap topology. Furthermore, it is a priori not clear what the right structure of the phase operator in the confined case is. Our calculation gives the answer in form of equation (13).

\section{Calculation of Friedel oscillations and boundary exponent}

In order to calculate the Friedel oscillations $\left\langle\delta \hat{\rho}_{F}(z)\right\rangle_{0}$ in the interacting ground state, we apply the Wick theorem in the well known form

$$
\left\langle e^{i \hat{\Phi}}\right\rangle=\exp \left(-\frac{1}{2}\left\langle\hat{\Phi}^{2}\right\rangle\right)
$$

utilizing the homogeneous structure of the phase operator $\hat{\Phi}$ equation (17). Thus

$$
\left\langle\cos 2\left[k_{F} \tilde{z}(z)-b\left(u_{0}(z)\right)+\hat{\Phi}\left(u_{0}(z)\right)\right]\right\rangle_{0}=\cos 2\left[k_{F} \tilde{z}(z)-b\left(u_{0}(z)\right)\right] e^{-2\left\langle\hat{\Phi}\left(u_{0}(z)\right)^{2}\right\rangle_{0}} .
$$

The mean square average with respect to the ground state becomes

$$
\left\langle\hat{\Phi}(u)^{2}\right\rangle_{0}=\sum_{m=1}^{\infty} \frac{\sin ^{2}(m u)}{m}\left(e^{-m \eta}+(K-1) e^{-m r}\right)
$$

which leads to

$$
\begin{aligned}
\left\langle\hat{\Phi}(u)^{2}\right\rangle_{0}=-\frac{1}{2} \ln \eta- & \frac{1}{2}(K-1) \ln r-\frac{1}{4} \ln [\mathcal{D}(2 u+i \eta) \mathcal{D}(-2 u+i \eta)] \\
& -\frac{1}{4}(K-1) \ln [\mathcal{D}(2 u+i r) \mathcal{D}(-2 u+i r)] .
\end{aligned}
$$

Here, the abbreviation

$$
\mathcal{D}(s) \equiv \frac{1}{1-e^{i s}}
$$

is introduced. Finally, one obtains 
$\exp \left\{-2\left\langle\hat{\Phi}(u)^{2}\right\rangle_{0}\right\}=\eta r^{K-1} 2^{-K / 2} \frac{\left[\left(1+e^{-2 r}\right) / 2-e^{-r} \cos (2 u)\right]^{(1-K) / 2}}{\left[\left(1+e^{-2 \eta}\right) / 2-e^{-\eta} \cos (2 u)\right]^{1 / 2}}$

Considering $r \ll 1, \eta \rightarrow 0+$, and

$$
\cos 2 u_{0}(z)=2\left(\frac{z}{L_{F}}\right)^{2}-1
$$

leads to the result for the Friedel oscillations in the limit $L_{F}-|z| \gg r^{2} L_{F} / 8$

$$
\left\langle\delta \hat{\rho}_{F}(z)\right\rangle_{0}=-\frac{(-1)^{N}}{2 \pi L_{F}}\left(\frac{r}{2}\right)^{K-1} \frac{\cos 2\left[k_{F} \tilde{z}(z)-b\left(u_{0}(z)\right)\right]}{Z(z)^{K+1}} .
$$

It is seen that attractive interactions, $K>1$, decrease the Friedel oscillations, while repulsive interactions, $K<1$, increase them at any fixed position $|z|<L_{F}$.

The backscattering phase shift in equation (35) is mostly a small correction as is seen by comparing equations (16) and (24), except when the coupling is exceedingly strong.

It is noted that the coupling constant $K$ goes to unity and $b\left(u_{0}(z)\right)$ to zero for vanishing interactions. This answers a question put forward in [25]: the divergence of the envelope near the boundaries for free Friedel oscillations in the harmonic trap has the conjectured boundary exponent $K_{0}=1$. For $|z| \ll L_{F}$, i.e., well inside the trap, one obtains the free Friedel oscillations

$$
\left\langle\delta \hat{\rho}_{F}(z)\right\rangle_{00}=-\frac{(-1)^{N}}{2 \pi L_{F}} \cos 2 k_{F} z
$$

in accordance with a corresponding result in [25], which was obtained by an asymptotic expansion of the exact particle density of non-interacting fermions.

Returning to the interacting case and specializing to the region near the classical boundaries, where

$$
Z^{2}(z) \rightarrow 2\left(1-|z| / L_{F}\right)
$$

the boundary exponent for the decay of Friedel oscillations away from these classical turning points is

$$
\nu=(K+1) / 2 .
$$


In contrast, the corresponding result for BLL in the case of one component is $\nu_{B L L}=K[15-17]$. The latter value is also obtained for an infinitely strong pinning impurity which acts as a hard wall [23], a case when pinning strength does not scale [24]. The present soft boundary thus causes a slower decay for repulsive interactions in comparison to the BLL while the situation is reversed for attractive interactions.

\subsection{Dependence on trap parameters}

We discuss the question how trap parameters influence the value of the boundary exponent and the amplitude of Friedel oscillations. In view of $k_{F}=\sqrt{m_{A} \omega_{\ell}(2 N-1) / \hbar}$ and equation (7), the central coupling constant $K$ according to equation (9) depends on longitudinal trap frequency $\omega_{\ell}$ and particle number $N$ (apart from interaction data). Two cases will be considered:

$\mathrm{i} \quad$ The trap is made shallower, i.e., $\omega_{\ell}$ is decreased keeping $\mathrm{N}$ constant. In this case, interactions become irrelevant and $K$ goes to unity. Because the amplitude in equation (35) is proportional to $1 / L_{F} \propto \sqrt{\omega_{\ell}}$ the Friedel oscillations vanish everywhere inside the huge trap as expected. However, they still increase towards the boundaries because the trap topology persists for all non-zero $\omega_{\ell}$.

ii A kind of thermodynamic limit [32] is defined by making $\omega_{\ell} \propto 1 / N$ and $N$ large such that the Fermi wave number and hence $\tilde{V}_{c}$ and $K \neq 1$ remain constant. Again, the prefactor in equation (35) suppresses the Friedel oscillations everywhere inside the trap. Interestingly, at large but finite $N$ the amplitude of Friedel oscillations increases faster towards the boundary when interactions are attractive in comparison to the repulsive case.

\section{Boundary exponents for two components}

We develop the corresponding theory for two components, e.g., two different hyperfine components of the same trapped fermionic atoms. We assume equal masses and equal trapping frequencies. The latter assumption is an approximation in the hyperfine case.

The local densities for mass and composition:

$$
\begin{aligned}
& \hat{\rho}(z) \equiv \sum_{m=0, n=0}^{\infty} \psi_{m}(z) \psi_{n}(z)\left[\hat{c}_{m+}^{+} \hat{c}_{n+}+\hat{c}_{m-}^{+} \hat{c}_{n-}\right] \\
& \hat{\sigma}(z) \equiv \sum_{m=0, n=0}^{\infty} \psi_{m}(z) \psi_{n}(z)\left[\hat{c}_{m+}^{+} \hat{c}_{n+}-\hat{c}_{m-}^{+} \hat{c}_{n-}\right]
\end{aligned}
$$

are treated as described in Section III. Two odd phase operators corresponding to $\hat{\phi}_{\text {odd }}$ appear. They are defined by 


$$
\hat{\phi}_{\sigma, \text { odd }}(u)=\frac{1}{2}\left(\hat{\phi}_{\sigma}(u)+\hat{\phi}_{\sigma}^{+}(u)-\hat{\phi}_{\sigma}(-u)-\hat{\phi}_{\sigma}^{+}(-u)\right)
$$

in terms of the two bosonization operators for the components $\sigma=1$ and $\sigma=-1$

$$
\hat{\phi}_{\sigma}(u)=-i \sum_{m=1} \frac{1}{\sqrt{m}} e^{i m(u+i \eta / 2)} \hat{b}_{m \sigma}
$$

The relation of the $\hat{b}$-operators to mass and composition fluctuation operators $\hat{d}$ is

$$
\hat{b}_{m \sigma}=\sum_{\nu} \frac{1}{\sqrt{2}} \sigma^{\frac{1-\nu}{2}} \hat{d}_{m \nu}=\frac{1}{\sqrt{2}}\left(\hat{d}_{m+}+\sigma \hat{d}_{m-}\right)
$$

The final representation of the total density operator is

$$
\begin{aligned}
\delta \hat{\rho}(z)=\frac{1}{\pi} \partial_{z}\left(\hat{\phi}_{+, \text {odd }}\left(u_{0}(z)\right)+\hat{\phi}_{-, \text {odd }}\left(u_{0}(z)\right)\right) & \\
& -\frac{(-1)^{N}}{\pi \eta L_{F} Z(z)}\left\{\cos \left[2 k_{F} \tilde{z}(z)+2 \hat{\phi}_{+, \text {odd }}\left(u_{0}(z)\right)\right]+\cos \left[2 k_{F} \tilde{z}(z)+2 \hat{\phi}_{-, \text {odd }}\left(u_{0}(z)\right)\right]\right\} .
\end{aligned}
$$

Defining the basic phase fields

$$
\hat{\Phi}_{\nu}(u) \equiv \frac{1}{\sqrt{2}}\left\{\hat{\phi}_{+, \text {odd }}(u)+\nu \hat{\phi}_{-, \text {odd }}(u)\right\}+b_{\nu}(u)
$$

with $\nu=1$ for mass and $\nu=-1$ for composition fluctuations, brings equation (43) into the form

$$
\begin{aligned}
\delta \hat{\rho}(z)=\frac{\sqrt{2}}{\pi} \partial_{z}\left(\hat{\Phi}_{1}\left(u_{0}(z)\right)-b_{1}\left(u_{0}(z)\right)\right) & \\
& -\frac{2(-1)^{N}}{\pi \eta L_{F} Z(z)} \cos \left[2 k_{F} \tilde{z}(z)+\sqrt{2}\left(\hat{\Phi}_{1}\left(u_{0}(z)\right)-b_{1}\left(u_{0}(z)\right)\right)\right] \cos \left(\sqrt{2} \hat{\Phi}_{-1}\left(u_{0}(z)\right)\right) .
\end{aligned}
$$

The analogue of equation (16) is

$$
b_{1}(u)=i \frac{K_{1} V_{c \|}}{4 \epsilon_{1}} \sqrt{2} \ln \left(\frac{1-e^{-r+2 i u}}{1-e^{-r-2 i u}}\right) .
$$

The quantity $b_{-1}$ vanishes identically. 
Coupling constants and renormalized level spacings are given by

$$
K_{m, \nu}=\sqrt{\frac{\left(\hbar \omega_{\ell}+V_{a \|}(m)+\nu V_{a \perp}(m)\right)-\left[V_{b \|}(m)+\nu V_{b \perp}(m)-V_{c \|}(m)\right]}{\left(\hbar \omega_{\ell}+V_{a \|}(m)+\nu V_{a \perp}(m)\right)+\left[V_{b \|}(m)+\nu V_{b \perp}(m)-V_{c \|}(m)\right]}},
$$

and

$\epsilon_{m, \nu}=\left[\left(\hbar \omega_{\ell}+V_{a \|}(m)+\nu V_{a \perp}(m)\right)^{2}-\left[V_{b \|}(m)+\nu V_{b \perp}(m)-V_{c \|}(m)\right]^{2}\right]^{1 / 2}$,

respectively. The subscript $\|$ refers to interactions between fermions of the same component while $\perp$ stands for different components [18].

Similarly, the composition operator reads as

$$
\begin{aligned}
\delta \hat{\sigma}(z)=\frac{\sqrt{2}}{\pi} \partial_{z} \hat{\Phi}_{-1}\left(u_{0}(z)\right) & \\
& +\frac{2(-1)^{N}}{\pi \eta L_{F}(z)} \sin \left[2 k_{F} \tilde{z}(z)+\sqrt{2}\left(\hat{\Phi}_{1}\left(u_{0}(z)\right)-b_{1}\left(u_{0}(z)\right)\right)\right] \sin \left(\sqrt{2} \hat{\Phi}_{-1}\left(u_{0}(z)\right)\right) .
\end{aligned}
$$

The central relation equation (15) generalizes to

$$
\begin{array}{r}
\hat{\Phi}_{\nu}(u) \equiv \sum_{n=1}^{\infty} \sqrt{\frac{1}{n}} e^{-n \eta / 2} \sin (n u)\left(\hat{d}_{n \nu}+\hat{d}_{n \nu}^{+}\right)+b_{\nu}(u) \\
=\sum_{n=1}^{\infty} \sqrt{\frac{K_{n \nu}}{n}} e^{-n \eta / 2} \sin n u\left(\hat{f}_{n \nu}+\hat{f}_{n \nu}^{+}\right) .
\end{array}
$$

In order to be able to evaluate the phase fluctuations in the way given above, i.e., for free phase fields, the interaction coefficient $V_{c \perp}$ for backward scattering between the two components must be zero. This is usually not the case. Relying on insight from the Luttinger model [21], one can expect that for $V_{c \|} \geq\left|V_{c \perp}\right|$ the coupling $V_{c \perp}$ scales to zero at low energies and that for $V_{c \|}=V_{c \perp}>0$ backscattering becomes irrelevant. In that case, $K_{-1}^{*}=1$ holds. This corresponds to spin isotropy in the Luttinger model. However, the present one-branch model hardly allows such a case in reality due to suppression of s-wave scattering in the parallel channel. With this provision, we give the results for two components based on the assumption that the field $\hat{\Phi}_{-1}(u)$ is a (renormalized) free field:

$\left\langle\delta \hat{\rho}_{F}(z)\right\rangle_{0}=-\frac{2(-1)^{N}}{\pi \eta L_{F} Z(z)} e^{-\left\langle\hat{\Phi}_{-1}^{2}\left(u_{0}(z)\right)\right\rangle_{0}} \cos \left(2 k_{F} \tilde{z}(z)-\sqrt{2} b_{1}\left(u_{0}(z)\right)\right) e^{-\left\langle\hat{\Phi}_{1}^{2}\left(u_{0}(z)\right)\right\rangle_{0}}$. 
Note that no Friedel oscillations exist in the composition part because $\langle\delta \hat{\sigma}(z)\rangle \equiv 0$.

With the renormalized value of the coupling constant $K_{-1} \rightarrow K_{-1}^{*}$, the Friedel oscillations are

$\left\langle\delta \hat{\rho}_{F}(z)\right\rangle_{0}=-\frac{(-1)^{N}}{\pi L_{F}} \cos \left[2 k_{F} \tilde{z}(z)-\sqrt{2} b_{1}\left(u_{0}(z)\right)\right]\left(\frac{r}{2}\right)^{\frac{K_{1}+K_{-1}^{*}}{2}-1} Z(z)^{-\left(\frac{K_{1}+K_{-1}^{*}}{2}+1\right)}$

giving a boundary exponent

$$
\nu=\frac{K_{1}+K_{-1}^{*}}{4}+\frac{1}{2}
$$

Again, this is different from the corresponding exponent

$$
\nu=\frac{K_{\rho}+K_{\sigma}^{*}}{2}
$$

of the BLL [15-17].

The result equation (53) also applies to spinfull one-dimensional fermions $(\nu=$ $1 \rightarrow c$ : charge degrees of freedom, $\nu=-1 \rightarrow s$ : spin degrees of freedom) and is thus applicable to harmonically trapped one-dimensional electrons.

\section{Summary}

We have calculated the quantum interference phenomenon of Friedel oscillations for the Tomonaga-Luttinger model of interacting one-dimensional fermionic atoms trapped in a harmonic potential. We used bosonization techniques and an asymptotic representation of the density operator in terms of a particular phase field. The result shows that the boundary exponent for the decay of Friedel oscillations away from the classical boundaries differs from the result for bounded Luttinger liquids where the fermions are confined between hard walls.

\section{Acknowledgments}

We gratefully acknowledge helpful discussions with F. Gleisberg and W. P. Schleich and financial help by Deutsche Forschungsgemeinschaft.

\section{References}

[1] DeMarco B and Jin D S 1999 Science 2851703 
[2] O'Hara K M 2000 et al Phys. Rev. Lett. 852092

[3] Schreck F et al 2001 Phys. Rev. A $64011402(\mathrm{R})$

[4] Truscott A G et al 2001 Science 2912570

[5] Schreck F et al 2001 Phys. Rev. Lett. 87080403

[6] Hadzibabic Z et al 2002 Phys. Rev. Lett. 88160401

[7] Hadzibabic Z et al 2003 cond-mat/0306050 v1

[8] Vuletic V et al 1998 Phys. Rev. Lett. 801634

[9] Fortagh J et al 1998 Phys. Rev. Lett. 815310

[10] Denschlag J, Cassettari D and Schmiedmayer J 1999 Phys. Rev. Lett. 822014

[11] Reichel J, Hänsel W and Hänsch T W 1999 Phys. Rev. Lett. 833398

[12] Ott H et al 2001 Phys. Rev. Lett. 87230401

[13] Leanhardt A E et al 2002 Phys. Rev. Lett. 89040401

[14] Friedel J 1958 Nuovo Cimento Suppl. 7287

[15] Fabrizio M and Gogolin A O 1995 Phys. Rev. B 5117827

[16] Wang Y, Voit J and Fu-Cho Pu 1996 Phys. Rev. B 548491

[17] Voit J, Yupeng Wang and Grioni M 2000 Phys. Rev. B 617930

[18] Wonneberger W 2001 Phys. Rev. A 63063607

Gao Xianlong and Wonneberger W 2002 ibid 65033610

Gao Xianlong et al 2003 ibid $\mathbf{6 7} 023610$

[19] Emery V J 1979 Highly Conducting One-Dimensional Solids ed J T Devreese et al (New York: Plenum) p 247

[20] Haldane F D M 1981 J. Phys. C: Solid State Phys. 142585

[21] Voit J 1995 Rep. Prog. Phys. 58977

[22] Schulz H J 1995 Mesoscopic Quantum Physics ed E Akkermans et al (Amsterdam: Elsevier) p 533

[23] Egger R and Grabert H 1995 Phys. Rev. Lett. 753505

[24] Kane C L and Fisher M P A 1992 Phys. Rev. B 461220

[25] Gleisberg F et al 2000 Phys. Rev. A 62063602

[26] Akdeniz Z et al 2002 Phys. Rev. A 66055601

[27] Gleisberg F and Wonneberger W 2002 cond-mat/0208376 v1

[28] Kronig de R L, Physica 19352968

[29] Regal C A et al 2003 Phys. Rev. Lett. 90053201

[30] Meden V et al 2000 Eur. Phys. J. B 16631

[31] Schönhammer K and Meden V 1996 Am. J. Phys. 641168

[32] Dalfovo F et al 1999 Rev. Mod. Phys. 71463 